\title{
Iatrogenic vertebral arteriovenous fistula
}

\author{
V I K I N G O L O V B J ÖR K \\ From the Department of Thoracic Surgery, University Hospital, Uppsala, Sweden
}

Carotid artery angiography is performed with increasing frequency. In centres where this investigation is commonly used the complications are negligible compared with the valuable information that can be obtained. But I have seen three cases in which a carotid angiography was complicated by a vertebral arteriovenous fistula. In one of these the symptoms were severe enough to indicate surgical treatment. The aim of this paper is to report this unusual technical procedure.

\section{CASE REPORT}

A 27-year-old woman had complained of intermittent headaches since childhood; the symptoms had got worse during the last year. Angiography of both carotid arteries was performed, but the quality of the angiogram obtained on the left side was not satisfactory and therefore a second carotid angiography was done. One day after the investigation the patient noticed a severe waterfall sound in her head and on auscultation a machinery murmur was heard under the left mandibular angle. She was sent to this hospital. On investigation we heard a loud machinery murmur along the left carotid artery, maximal at the level of the larynx. There was no thrill. A thoracic aortography was performed as well as a left vertebral angiography, and a diagnosis of arteriovenous fistula involving the left vertebral artery was confirmed. Furthermore the left vertebral artery was three times the size of the right vertebral artery. At the level of the fourth cervical vertebra contrast was seen passing directly from the vertebral artery to the veins within the vertebral canal. Selective catheterization of the left vertebral artery confirmed the diagnosis with a fistula at the level of the fourth cervical vertebra. The patient could not tolerate this continuous murmur, which was disturbing her day and night, and an operation was therefore performed.

An incision was made along the anterior end of the sternocleidomastoideus muscle on the left side. The transverse process was exposed behind the carotid artery, and the cervical muscles were dissected away from the vertebral body. The arterial vertebral canal was identified in three vertebrae corresponding to the fifth, fourth, and third cervical vertebrae; in other words, one above, one at the level of, and one below the angiographic demonstration of the fistula. The vertebral canal was opened by removing the overlying bone. After liberation of the vertebral artery and vein it was impossible to dissect the artery and vein free and to localize the fistula itself for suture, as had been planned. The artery was surrounded by a paper-thin venous plexus; the veins were so thin that they could only be sutured with 6-0 silk. The vertebral artery and its vein were ligated at the level of the third, fifth, and sixth cervical vertebrae (see Fig. 1). The wound was closed and the murmur disappeared.

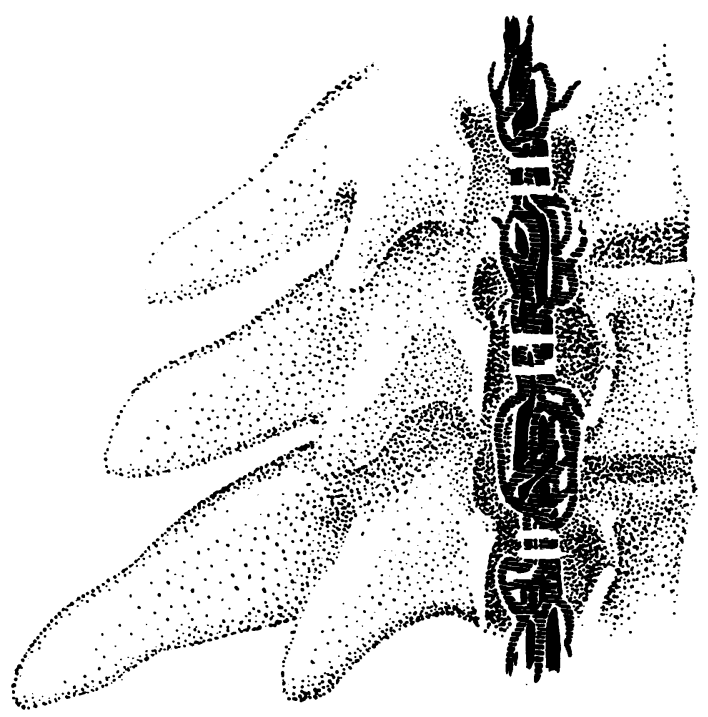

FIG. 1. The vertebral canals and three opened vertebrae. The vertebral artery and veins are ligated at three different levels, the third, fifth, and sixth cervical vertebrae.

The patient made an immediate uneventful recovery except for some weakness in the deltoid muscle which gradually disappeared. On the tenth day after operation she developed a sudden headache and vomiting and complained of difficulty in reading. Her vision was normal; neurological investigation suggested a spreading thrombosis from the ligated vertebral artery with impairment of the basilar circulation. Anticoagulant treatment was started. The symptoms regressed after one week and the patient was able to leave hospital. One year after the operation she was in very good condition. There was no murmur, but 
the patient complained of weakness of the right hand when writing. She has not returned to work because she is pregnant.

\section{DISCUSSION}

Ligation of the vertebral artery only at its origin would probably not have prevented a recurrence of the murmur. Therefore it was decided to open the vertebral canal and ligate the artery and vein both above and below the arteriovenous communication. This was done and the murmur disappeared.

It is evident that the amount of blood shunted through this small fistula was not significant. But the murmur that disturbed the patient day and night was significant, and when this became intolerable operation was advised. But it is evident that the operation is of such magnitude that, unless the patient insists, it should not be performed.

A second patient who had the same symptoms of a fistula between the vertebral arteries and veins was seen later. In this patient the machinery murmur was found accidentally and the patient had no complaints. Operation was not advised.

A third patient, 51 years old, developed a $\frac{\omega}{\sigma}$ similar continuous murmur after angiography. As $\propto$ her dominating symptoms were dizziness, headaches, and difficulty in walking after a head $\vec{\circ}$ trauma with unconsciousness, surgery for the vertebral fistula was not advised.

I suggest that a characteristic clinical sign of an arteriovenous fistula that involves the vertebral $\vec{x}$ vessels is that although a very loud machinery murmur can be heard no thrill can be palpated. A fistula that involves the carotid artery usually causes a thrill.

\section{SUMMARY}

Surgical treatment of a case of iatrogenic arteriovenous vertebral fistula is discussed. Operation is advised only if the patient has intractable symptoms. 